\title{
Sera from patients with motor neuron disease and associated paraproteinaemia fail to inhibit experimentally induced sprouting of motor nerve terminals
}

\author{
MICHAEL DONAGHY, LW DUCHEN \\ From the Departments of Neurology and Neuropathology, Institute of Neurology and The National Hospital for \\ Nervous Diseases, London, $U K$
}

SUMMARY IgG kappa paraproteinaemia was discovered in one patient with amyotrophic lateral sclerosis and one with chronic spinal muscular atrophy. Serum from these patients was injected into mouse muscles paralysed by botulinum toxin which is known to induce sprouting from motor nerve terminals. Daily injection of serum for 7 days failed to inhibit terminal sprouting. It is concluded that the paraproteins did not recognise a growth factor postulated as being implicated in the pathogenesis of motor neuron disease.

Injection of botulinum toxin (BoTx) into skeletal muscle causes motor nerve terminals to sprout. ${ }^{1}$ Such sprouting has been reported to be inhibited by subsequent injection of serum from patients with amyotrophic lateral sclerosis. ${ }^{2}$ It is possible that disorders of neuromuscular connectivity could be caused by autoantibodies to a growth factor which is normally secreted by muscle fibres and controls their innervation. A rare clinical association exists between paraproteinaemia and amyotrophic lateral sclerosis, spinal muscular atrophy or motor neuropathy. ${ }^{3}$ In such patients the paraprotein might possess autoantibody activity against a motor nerve growth factor. We have tested paraproteinaemic sera from two patients with disordered neuromuscular connectivity for their capacity to inhibit motor nerve terminal sprouting induced by BoTx in mouse soleus muscle.

\section{Methods}

Subjects Monoclonal gammopathies were detected by routine serum electrophoresis in two patients under investigation for neuromuscular disease. Fresh serum from these patients and a healthy human control without para-

Address for reprint requests: Dr M Donaghy, National Hospital for Nervous Diseases, Queen Square, London WCIN 3BG, UK.

Received 5 November 1985. Accepted 23 November 1985 proteinaemia were stored frozen in $0.3 \mathrm{ml}$ aliquots at $-20^{\circ} \mathrm{C}$

Animal experiments CFLP mice weighing 25-35g were used. A single dose of $0.004 \mu \mathrm{g}$ type A BoTx (Sigma) in $0.1 \mathrm{ml}$ gelatin phosphate buffer was injected deep into the calf muscles of the right leg. Two hours later, and daily thereafter, $0 \cdot 1 \mathrm{ml}$ of serum was injected into the same site. Two animals were given BoTx alone, and the patients' or control sera were each given to four animals. All mice developed profound weakness of the right leg. After 7 days the mice were killed by intracardiac perfusion with $1 \%$ calcium acetate in $10 \%$ formalin under ether anaesthesia. The soleus muscle was immediately dissected out and fixed for 1 hour in calcium formalin solution at $4^{\circ} \mathrm{C}$ before sectioning. It is known from previous work that sprouting from motor endplates is well established 7 days after BoTx injection into mouse soleus muscle. ${ }^{4}$

Histology Serial longitudinal $20 \mu \mathrm{m}$ frozen sections of the muscle were stained for cholinesterase activity and nerve fibres. ${ }^{5}$ Occasional sections were distorted or lost during sectioning. Slides were coded and subsequently examined "blind". Each motor end-plate was examined under high power and, if a preterminal axon was identified, the presence and number of terminal sprouts were recorded.

\section{Case histories}

Patient 1 A 72-year-old woman had progressive weakness of the legs and right hand for 9 months. Examination revealed widespread weakness and fasciculations in all four limbs with hyperreflexia, an extensor left plantar response 
and no sensory loss. Electromyography showed partial denervation of the limb muscles with normal sensory nerve conduction. Myelography and spinal fluid examination were normal. Her limbs weakened progressively with marked wasting of the small muscles of the hand and she developed dysphagia and wasting of the tongue. Serum electrophoresis revealed a paraprotein in the gamma region which was characterised as IgG kappa at a concentration of $4 \mathrm{~g} / \mathrm{l}$. The clinical diagnosis was amyotrophic lateral sclerosis.

Patient 2 This 59-year-old man had initially noticed difficulty in standing on tiptoe 20 years previously. Since then the legs had become progressively weaker with wasting and fasciculation. His hand grip had been weak for 1 year. Examination revealed slightly weak small hand muscles, generalised weakness and wasting of both legs, more marked distally, absent ankle jerks but otherwise normal tendon reflexes and normal sensation. Electromyography demonstrated denervation of the proximal and distal muscles of both the arms and legs. Motor and sensory nerve conduction were normal except for reduced motor conduction velocity to extensor digitorum brevis which was associated with small muscle action potentials and attributed to loss of large diameter motor axons. Serum electrophoresis detected a paraprotein in the gamma region subsequently characterised as IgG kappa at a concentration of $6.5 \mathrm{~g} / \mathrm{l}$. Bone marrow examination, radiological skeletal survey and urine testing for Bence-Jones protein were all normal. There was no family history of neuromuscular disease. The clinical diagnosis was chronic spinal muscular atrophy of sporadic or autosomal recessive type.

\section{Results}

The histological appearance of sprouting nerve terminals is shown in the figure. End-plates were readily identified by the dense concentration of cholinesterase reaction product. Only end-plates whose preterminal axon was identifiable were included in this quantitative survey. At some end-plates, no sprouts could be seen in direct continuity with the end-plate but at others delicate axonal sprouts could be seen arising from terminals within the end-plates. These sprouts could be readily distinguished from the preterminal myelinated fibre by their calibre and the common occurrence of crystals of cholinesterase reaction product along them.

The table shows the proportion of end-plates from which sprouts were arising 7 days after injection with BoTx alone, BoTx and control serum, and BoTx and paraproteinaemic serum from patient 1 or patient 2 . Although the proportion of terminals which had sprouted was similar in all groups, the muscles injected with patients' sera contained a slightly larger number of sprouts per end plate than the control
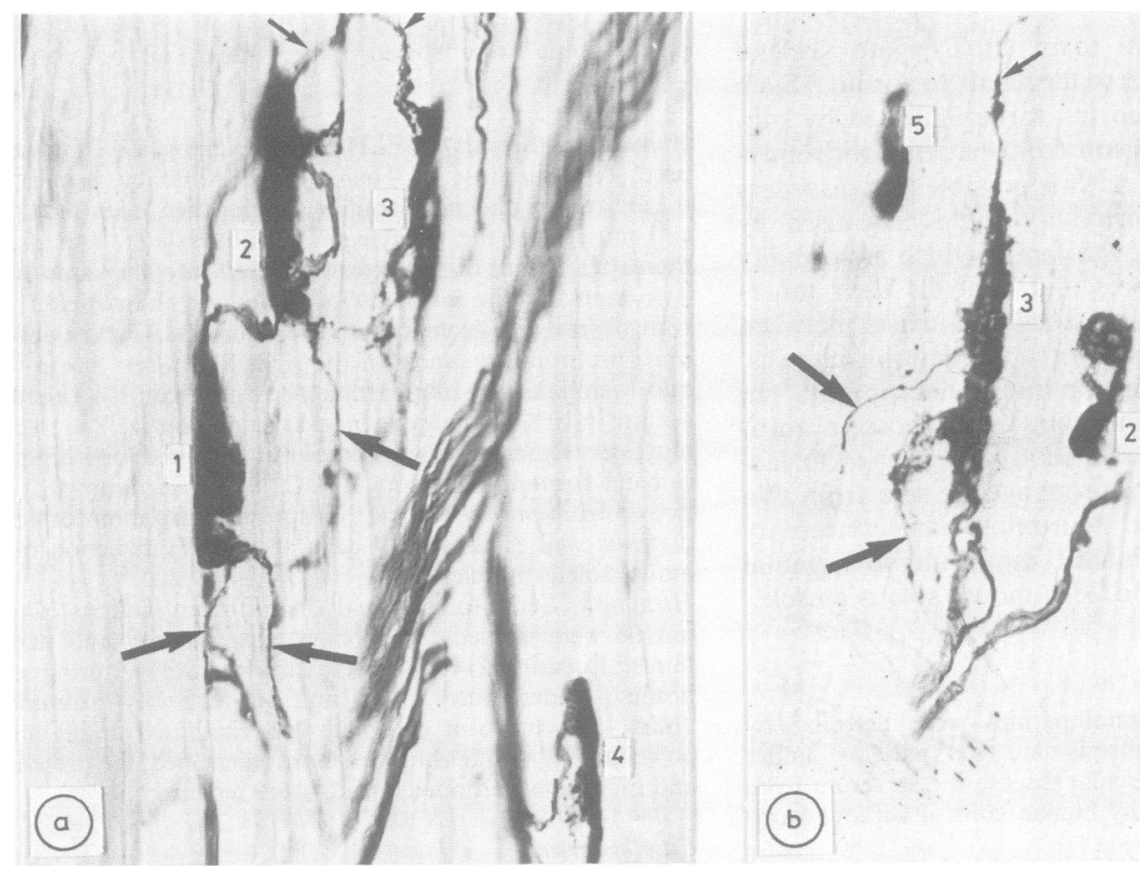

Fig Frozen sections of mouse soleus stained to show end-plate cholinesterase and nerve fibres 7 days after injection of $(A)$ BoTx and serum from patient with amyotrophic lateral sclerosis $(B)$ BoTx and control serum. In fig $1(A)$ end-plates numbered 1,2 , and 3 show terminal sprouting whereas 4 does not. In fig $1(B)$ only end-plate 3 shows sprouts, 1 and 2 do not, and 4 and 5 were not included in the counts since their preterminal axons were not seen. (Arrows to some sprouts) $(\times 500)$ 
Table Proportion of mouse soleus end-plates with ultraterminal sprouts 7 days after BoTX

\begin{tabular}{lcc}
\hline Experimental group & $\begin{array}{l}\text { Total end-plates } \\
\text { counted }\end{array}$ & $\%$ Sprouting \\
\hline BoTx alone & $366(2)$ & $56 \cdot 7$ \\
BoTx + control serum & $656(4)$ & $58 \cdot 9$ \\
BoTx + patient 1 serum & $1102(4)$ & $61 \cdot 1$ \\
BoTx + patient 2 serum & $284(4)$ & 60.9 \\
\hline The numbers in brackets indicate the number of muscles studied
\end{tabular}

The numbers in brackets indicate the number of muscles studied

muscle. These results show that the two paraproteinaemic sera from patients with disorders of neuromuscular connectivity did not inhibit motor nerve terminal sprouting in this test system.

\section{Discussion}

This experimental study was prompted by the possibility that circulating IgG kappa paraproteins were of pathogenetic importance to the disorder of neuromuscular connections seen in one patient with amyotrophic lateral sclerosis and another with spinal muscular atrophy. We postulated that the circulating monoclonal immunoglobulins in these patients might possess autoantibody activity against a motor nerve terminal growth factor secreted by skeletal muscle, or its receptor. Our results did not support this notion.

Evidence has been presented that skeletal muscle fibres control their innervation by secreting a motor nerve terminal growth factor (reviewed in ref 6). Motor terminals sprout in response to presynaptic blockade with BoTx, ${ }^{1}$ postsynaptic blockade with $\alpha$-bungarotoxin ${ }^{7}$ or tetrodotoxin blockade of motor axons. ${ }^{8}$ Sprouting can be prevented by implanting an additional nerve ${ }^{9}$ or by direct electrical stimulation of muscle $^{10}$ implying that the sprouting stimulus comes from inactive muscle fibres. Denervated rat diaphragm secretes a 56000 Dalton MWt protein and antisera recognising this protein have been reported to inhibit motor nerve terminal sprouting induced by BoTx in mouse gluteus muscle. ${ }^{11}$ Gurney and his collaborators have provided evidence that autoantibodies recognising this putative motor nerve growth factor might be of pathogenetic significance in some cases of human motor neuron disease. ${ }^{2}$ Sera from four out of 19 patients with sporadic amyotrophic lateral sclerosis inhibited BoTx-induced motor nerve terminal sprouting to a greater degree than did sera from healthy human controls, diabetics with neuropathy, patients with familial amyotrophic lateral sclerosis or if animals were injected with BoTx alone. Immunoblotting methods were used to show that immunoglobulin in some of the sprout-inhibiting amyotrophic lateral sclerosis sera recognised the 56000 Dalton MWt protein which is the putative motor terminal sprouting factor.
Various explanations are possible for the failure of paraproteinaemic sera to suppress motor nerve terminal sprouting in our experiments. The paraproteinaemia might be completely unrelated to the patient's neuromuscular disease, or it might have caused the neuromuscular disease by a mechanism unrelated to the trophic system responsible for motor nerve terminal sprouting. It is also possible that the paraprotein is a monoclonal immunoglobulin which recognises determinants which are present on motor nerve growth factor or its receptor in man but not mice.

We thank Miss Patricia Carter for the histological preparations and Dr CJ Earl and Dr P Rudge for permission to report on patients under their care.

\section{References}

${ }^{1}$ Duchen LW, Strich SJ. The effects of Botulinum toxin on the pattern of innervation of skeletal muscle in the mouse. $Q$ Jl Exp Physiol 1968;53:84-9.

${ }^{2}$ Gurney ME, Belton AC, Cashman N, Antel JP. Inhibition of terminal axonal sprouting by serum from patients with Amyotrophic Lateral Sclerosis. $N$ Engl J Med 1984;311:933-9.

${ }^{3}$ Latov N. Plasma cell dyscrasia and motor neuron disease. In: Rowland LP, ed. Human Motor Neuron Diseases. New York: Raven Press. 1982:273-8.

${ }^{4}$ Duchen LW. Changes in motor innervation and cholinesterase localisation induced by botulinum toxin in skeletal muscle of the mouse: differences between fast and slow muscle. J Neurol Neurosurg Psychiatry 1970; 33:40-54.

${ }^{5}$ Namba T, Nakamura T, Grob D. Staining for nerve fibre and cholinesterase activity in fresh frozen sections. $\mathrm{Am}$ $J$ Clin Pathol 1967;47:74-7.

${ }^{6}$ Slack Jr, Hopkins WG, Pockett S. Evidence for a motor nerve growth factor. Muscle Nerve 1983;6:243-52.

${ }^{7}$ Holland RL, Brown MC. Postsynaptic transmission block can cause motor nerve terminal sprouting. Science 1980;207:649-51.

${ }^{8}$ Brown MC, Ironton R. Motor neurone sprouting induced by prolonged tetrodotoxin block of nerve action potentials. Nature 1977;265:459-61.

${ }^{9}$ Duchen LW, Tonge DA. The effects of implantation of an extra nerve on axonal sprouting usually induced by botulinum toxin in skeletal muscle of the mouse. $J$ Anat 1977;124:205-15.

${ }^{10}$ Brown MC, Holland RL, Ironton R. Nodal and terminal sprouting from motor nerves in fast and slow muscles of the mouse. J Physiol (Lond) 1980;306:493-510.

${ }^{11}$ Gurney ME. Suppression of sprouting at the neuromuscular junction by immune sera. Nature 1984;307: 546-8. 